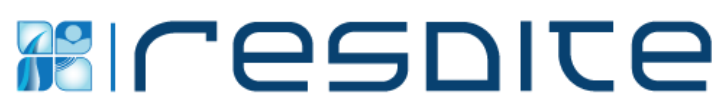

\section{Levantamento e avaliação de aplicativos sobre ansiedade e depressão disponíveis em língua portuguesa}

\author{
SURVEY AND EVALUATION OF ANXIETY AND DEPRESSION APPLICATIONS AVAILABLE IN \\ PORTUGUESE
}

Josefa de Jesus Querino', Natália Nascimento Andrade², Grazielle Batista dos Santos ${ }^{3}$, Lidiany Cerqueira Santos ${ }^{4}$

1 Graduanda em Farmácia. Universidade Federal de Sergipe.

ORCID: https://orcid.org/0000-0001-8204-1305

Email: josefa.barril13@gmail.com

2 Graduanda em Farmácia. Universidade Federal de Sergipe.

ORCID: https://orcid.org/0000-0003-0523-9494

Email: naataaliaa.na@hotmail.com

3 Graduanda em Fisioterapia. Universidade Federal de Sergipe.

ORCID: https://orcid.org/0000-0001-5074-1980

Email: graziellebatista2014@gmail.com

4 Mestre em Ciência da Computação. Universidade Federal de Sergipe.

ORCID: https://orcid.org/0000-0002-4989-0986

Email: lidianycs@ufs.br

Correspondência: Universidade Federal de Sergipe. Avenida Governador Marcelo Déda, 13, Centro, Lagarto - SE. Brasil. CEP 49400-000.

Copyright: Esta obra está licenciada com uma Licença Creative Commons Atribuição-NãoComercial 4.0 Internacional.

Conflito de interesses: os autores declaram que não há conflito de interesses.

\section{Como citar este artigo}

Querino J de Jesus; Andrade NN; Santos GB dos; Santos LC. Levantamento e avaliação de aplicativos sobre ansiedade e depressão disponíveis em língua portuguesa. Revista de Saúde Digital e Tecnologias Educacionais. [online], volume 5, n. 2. Editor responsável: Luiz Roberto de Oliveira. Fortaleza, julho de 2020, p. 72-88. Disponível em: http://periodicos.ufc.br/resdite/index. Acesso em "dia/mês/ano".

Data de recebimento do artigo: $16 / 12 / 2019$

Data de aprovação do artigo: 14/05/2020

Data de publicação: $20 / 07 / 2020$

\section{Resumo}

Objetivo: Realizar um levantamento e uma avaliação dos aplicativos sobre ansiedade e depressão em Língua Portuguesa, nas plataformas Android e iOS. Método: Trata-se de uma pesquisa exploratória, descritiva, em que se realizou uma busca nessas plataformas entre 20 de agosto e 6 de setembro de 2019, com o uso dos termos "ansiedade e depressão". Os aplicativos foram catalogados e selecionados de acordo com os critérios de inclusão definidos (atualizados há pelo menos um ano, em Língua Portuguesa). Foi usado como base um framework para avaliação de aplicativos de saúde mental. Resultados: Foram encontrados 274 aplicativos, entre os quais, 57 foram avaliados, sendo 53 para Android, 3 para iOS e 1 multiplataforma. Em relação ao custo, apenas $5 \%$ não eram totalmente gratuitos. A maioria dos aplicativos para iOS foi excluída por estarem disponíveis somente em inglês. Conclusão: Os aplicativos de saúde mental têm potencial para alcançar uma grande parcela da população, sendo uma estratégia promissora para diminuir a incidência e prevalência da ansiedade e depressão. Na avaliação, foram observados 
problemas como falta de acessibilidade, de política de privacidade, indicação de fontes de informação e evidências científicas que comprovem a sua eficácia, apesar disso, os aplicativos apresentaram boa experiência de uso.

Palavras-Chave: Aplicativos Móveis.

Ansiedade. Depressão.

\begin{abstract}
Objective: Conduct a survey and an evaluation of applications on anxiety and depression in Portuguese, on Android and iOs platforms. Method: This is an exploratory, descriptive research, in which a search was carried out on these platforms between August 20 and September 6, 2019, using the terms "anxiety and depression". The applications were cataloged and selected according to the defined inclusion criteria (updated at least one year ago, in
\end{abstract}

Portuguese). A framework for evaluating mental health applications was used as the basis. Results: 274 applications were found, of which 57 were evaluated, 53 for Android, 3 for iOS and 1 multiplatform. Regarding the cost, only 5\% were not completely free. Most iOS applications have been excluded because they are only available in English. Conclusion: Mental health applications have the potential to reach a large portion of the population, being a promising strategy to decrease the incidence and prevalence of anxiety and depression. In the evaluation, problems were observed such as lack of accessibility, privacy policy, indication of information sources and scientific evidence that prove its effectiveness, despite that, the applications presented good experience of use.

Keywords: Mobile Applications. Anxiety. Depression.

\section{Introdução}

De acordo com a Organização Mundial da Saúde (OMS), problemas de saúde mental são as principais causas de morbidade nas sociedades atuais ${ }^{1}$. Os distúrbios mentais mais comuns prevalentes em grande parte da população são divididos em duas principais categorias de diagnóstico: transtornos de ansiedade e transtornos depressivos ${ }^{2}$. Esses transtornos são caracterizados por diversas alterações nas funções psíquicas e podem provocar sintomas como tristeza, perda de interesse ou prazer e ainda sentimento de culpa ou baixa autoestima' ${ }^{1}$ São sintomas que, dependendo do grau, podem afetar a qualidade de vida de uma pessoa ${ }^{3}$.

Nos últimos anos, a alta incidência de diagnóstico da ansiedade e depressão na população em geral tornou-se uma questão preocupante ${ }^{4,5}$. Estima-se que, no mundo, 250 milhões de pessoas de todas as faixas etárias sofram de depressão, sendo a quarta doença mais diagnosticada. Esse número é muito alto e representa grande parte da população mundial que, atualmente, é de cerca de 7,5 bilhões $^{6}$. No Brasil, a depressão atinge 11,5 milhões de pessoas (5,8\% da população), enquanto distúrbios relacionados à ansiedade afetam mais de 18,6 milhões de brasileiros (9,3\% da população) ${ }^{7}$.

A falta de diagnóstico/tratamento dessas condições pode levar ao sofrimento psíquico e somático, discriminação, isolamento social, interrupção ou diminuição do rendimento dos estudos/trabalho, abuso de drogas/álcool e aumento da mortalidade 
(suicídio, homicídio) ${ }^{8}$. É, portanto, necessária a realização de rastreamento desses problemas mentais, para auxiliar na busca por diagnóstico correto, bem como uma intervenção adequada?

Sabe-se que a população adota e busca cada vez mais aplicativos de celulares para gerenciar sua saúde e rotina de vida ${ }^{10}$. O amplo acesso a smartphones e conectividade a aplicativos resultou em inovações baseadas em tecnologias móveis na área da Saúde e está resultando em uma área emergente denominada saúde móvel ( $m$-health), que se refere à oferta de serviços médicos e/ou de Saúde Pública que utilizam o apoio tecnológico de dispositivos móveis, como telefones celulares, computadores, sensores e outros dispositivos diretamente conectados ao usuário ${ }^{11,12}$.

A Food and Drug Administration (FDA) dos Estados Unidos estima que, em 2018, 1,7 bilhão de pessoas baixou aplicativos móveis de Saúde ${ }^{11}$. Ainda nos EUA, $85 \%$ dos jovens possuem smartphone e três quartos deles os utilizam para acessar informações de Saúde ${ }^{13}$. Dentre esses, $80 \%$ dos que tinham até 45 anos de idade demonstraram desejo em utilizar aplicativos móveis para rastrear sua saúde mental ${ }^{13}$. Reconhece-se que, para alguns estudos de saúde mental, a capacidade dos aplicativos de fornecer informações e intervenções clínicas é considerada uma grande vantagem, já que estes têm potencial de atingir várias pessoas através dos dispositivos eletrônicos ${ }^{13}$.

Segundo dados divulgados pela Agência Nacional de Telecomunicações (Anatel), em 2014, já haviam mais celulares do que pessoas no mundo, com aproximadamente 273,58 milhões de aparelhos. O Brasil terminou o mês de maio de 2017 com 242,1 milhões de celulares, sendo este o equipamento mais usado para acessar a internet no país ${ }^{14}$. Em 98,7\% dos domicílios com acesso à internet, o telefone móvel celular era utilizado para esse fim $^{14}$.

Contudo, apesar do crescimento no acesso à tecnologia e do interesse da popularização dos aplicativos de Saúde, foi identificada a falta de trabalhos que avaliem os aplicativos para dispositivos móveis disponíveis em Língua Portuguesa. Diante dessa lacuna, considerando o potencial dos aplicativos para a Saúde Pública, o presente estudo tem como objetivo realizar um levantamento e avaliação dos aplicativos sobre ansiedade e depressão em Língua Portuguesa, nas plataformas Android e iOS.

\section{Métodos}

Este trabalho consiste em um estudo exploratório e descritivo no qual foram realizados o levantamento e a avaliação de aplicativos sobre ansiedade e depressão. Para 
a condução da pesquisa, foi utilizado um protocolo com base em um método de revisão quasi-sistemática de literatura ${ }^{15,16}$. Tal protocolo foi adaptado e utilizado com o intuito de realizar uma pesquisa estrita e bem definida, com base em uma sequência organizada de fases, buscando-se produzir resultados mais confiáveis ${ }^{17}$.

O protocolo adotado para a realização desta pesquisa seguiu um modelo baseado em três fases (figura 1, nos anexos), detalhadas nas próximas subseções.

\section{Fase de Planejamento}

Neste primeiro momento, foram estabelecidas as necessidades e o direcionamento da pesquisa. Foram definidos o objetivo, as questões de pesquisa, a validação do escopo de pesquisa, um protocolo de busca e seleção dos aplicativos a serem avaliados. 0 protocolo especificou as fontes de busca, a metodologia utilizada e os critérios para a inclusão ou exclusão dos aplicativos encontrados. Para o levantamento dos aplicativos, foram definidas como bases de busca a Play Store e App Store, através das respectivas plataformas Android e iOS. Os termos utilizados nas buscas foram: "depressão e ansiedade" e somente "depressão ansiedade". Esses termos foram escolhidos com base no consenso entre os autores.

\section{Fase de Condução}

Essa etapa se inicia com a execução da busca pelos termos definidos na fase de planejamento. Os aplicativos encontrados foram analisados e avaliados com base no escopo de pesquisa e nos critérios estabelecidos. A busca pelos aplicativos foi conduzida entre 20 de agosto e 6 de setembro de 2019. Foram definidos os seguintes critérios de inclusão: aplicativos gratuitos ou com versão gratuita para testes, sobre ansiedade e depressão, disponíveis em Língua Portuguesa e com atualização recente. Foram excluídos os aplicativos que não estavam totalmente em português, que apresentavam somente uma versão paga e que não foram atualizados há pelo menos um ano da data de avaliação.

Os parâmetros definidos para a avaliação dos aplicativos encontrados foram baseados na lista de estruturas de avaliação dos aplicativos em Saúde desenvolvida nos estudos de ${ }^{11} \mathrm{e}^{20}$. Essa lista de estruturas de avaliação apresentada na Figura 2 (nos anexos) foi desenvolvida de acordo com níveis de prioridade e foram considerados aspectos como custo, publicidade, segurança e privacidade dos dados do usuário, eficácia do aplicativo, integração clínica, entre outros. Os autores realizaram a avaliação dos aplicativos após a 
instalação e utilização. A avaliação foi realizada de forma independente e as discordâncias foram discutidas até a obtenção de um consenso.

\section{Relatório da Avaliação}

Nesta última fase, foram discutidos os dados obtidos nas fases anteriores e expostas as ações tomadas para mitigar as ameaças à validade na avaliação.

A partir da lista de critérios estabelecidos na fase anterior para a avaliação dos aplicativos encontrados, foi criada uma tabela com todos os parâmetros a serem analisados, obtendo assim, no final, uma tabela completa com os aplicativos avaliados (tabela 1, nos anexos).

Após as buscas, foram encontrados 246 aplicativos na Play Store e 28 na App Store. Foram incluídos, na avaliação, os aplicativos gratuitos ou com versão gratuita para testes sobre ansiedade e depressão, disponíveis em Língua Portuguesa e com atualização recente. Aplicativos que não estavam totalmente em português, pagos e que não foram atualizados há pelo menos um ano da data de avaliação, foram excluídos do estudo. No final, obteve-se uma amostra composta por 54 aplicativos para o sistema Android e quatro para o iOS. 0 aplicativo “Cíngulo: Terapia Guiada" foi encontrado em ambos os sistemas, totalizando 57 aplicativos avaliados. Os aplicativos "Meditopia: meditação, ansiedade”, “Cíngulo: Terapia Guiada" e "Zen" apresentam uma versão gratuita para testes por 7 dias que foi utilizada para avaliação.

\section{Resultados}

Considerando as plataformas Android e iOS, foram avaliados 57 aplicativos. A Tabela 1 apresenta os resultados da avaliação.

Os aplicativos foram avaliados em termos de estabilidade, credibilidade, custos, privacidade, segurança, coleta de dados, uso de internet, base científica, facilidade de uso e acessibilidade ${ }^{11,20}$.

Em relação aos custos, dos 57 aplicativos avaliados, apenas 5\% não eram totalmente gratuitos, fato que facilita o acesso da população de países como o Brasil a esse tipo de ferramenta. Entretanto, apesar de a maioria dos aplicativos analisados ser gratuita, a necessidade de conexão com a internet para que o usuário possa utilizar o aplicativo foi de $78,9 \%$, o que pode restringir o uso. Outro ponto avaliado foi a presença de anúncios, 
encontrados em $87,7 \%$ dos aplicativos avaliados, o que pode tornar a experiência de uso lenta e fragmentada.

Outro parâmetro avaliado foi o conteúdo disponibilizado nos aplicativos. Foi verificado se esses fornecem informações exatas, precisas e baseadas em evidências científicas. Entre os avaliados, $59,6 \%$ destes não apresentavam informações com referências científicas. Isso demonstra que os usuários estão expostos ao risco de obter informações que não podem ser consideradas confiáveis. Em geral, os aplicativos de saúde mental disponíveis no mercado oferecem poucas informações sobre a qualidade do conteúdo apresentado, sendo poucos os que citam a fonte desse conteúdo e que fundamentam sua eficácia ${ }^{12}$. Dessa forma, é visível a possibilidade de exposição dos usuários a conteúdos de baixa qualidade científica, sendo necessários alguns ajustes para ampliar a eficácia dos recursos e fidedignidade das informações para os usuários.

Nesse sentido, além do embasamento teórico e científico no desenvolvimento dos aplicativos, é fundamental que eles sejam testados quanto à sua usabilidade e eficácia, já que, se desenvolvidos adequadamente, atingem grande público e passam a representar uma ferramenta importante de Saúde Pública ${ }^{23}$. 0 avanço no uso de tecnologias móveis como os aplicativos de smartphones tem sido considerado uma estratégia eficaz na produção de ferramentas com intervenções baseadas em evidências que reduzam riscos, ampliem acesso a serviços e melhoras na inclusão e interatividade social ${ }^{10,20}$. Ademais, os aplicativos auxiliam no desenvolvimento e na disseminação das informações de educação em saúde, contribuindo para a promoção da saúde dos usuários ${ }^{20,21}$.

Outro aspecto avaliado foi a acessibilidade dos aplicativos para pessoas com deficiência. A acessibilidade significa que páginas da web, ferramentas e tecnologias devem ser projetadas de forma que pessoas com deficiência possam utilizá-las ${ }^{21}$. 0 uso de dispositivos móveis por pessoas com deficiência tem aumentado consideravelmente e aberto inúmeras possibilidades de uso ${ }^{21}$. No entanto, durante a avaliação dos aplicativos, identificou-se que apenas um deles era acessível para pessoas com deficiência, demonstrando a falta de acessibilidade na maioria dos aplicativos encontrados. Assim, destaca-se a necessidade do desenvolvimento de aplicativos acessíveis para as pessoas com deficiência.

Entre os parâmetros avaliados, verificou-se também a possibilidade de excluir a conta e os dados, após a utilização do aplicativo. Foi observado que apenas 10,5\% dos aplicativos avaliados não apresentavam este recurso, ou seja, a maioria permite que o usuário cancele 
sua conta, caso queira desinstalar o aplicativo porque não gostou ou não achou relevante. Este é um recurso importante, uma vez que confere comodidade e segurança aos usuários, permitindo que seja possível cancelar a conta a qualquer momento.

Também foi avaliada a existência de política de privacidade. Essa política serve como principal meio de comunicação com o usuário dos aplicativos, informando quais são e como as informações pessoais confidenciais são utilizadas ${ }^{22}$. Em meio aos aplicativos avaliados, $42,1 \%$ não apresentam uma política de privacidade, não garantindo assim a segurança, a credibilidade e transparência dos dados aos seus usuários. Apesar de ser um documento jurídico importante, não existe um formato padrão dessas políticas para apresentar a informação, ou seja, a linguagem, os detalhes e as informações contidas nelas podem variar de acordo com os aplicativos. Ademais, a maioria delas é difícil de entendimento pelos usuários, pois apresentam diferentes interpretações, de modo que, mesmo com preocupações quanto a suas informações pessoais, muitos usuários ignoram a leitura dos termos ${ }^{22}$.

Com relação à avaliação da usabilidade, foi verificado se os aplicativos instalam, carregam, executam e são fáceis de usar, de maneira que forneçam uma experiência razoável ao usuário, causando uma boa impressão. A maioria demonstrou facilidade de uso, sendo possível utilizá-los de forma adequada com uma boa experiência. Ademais, é importante ressaltar que a usabilidade do aplicativo consiste em avaliar se o produto a ser utilizado por usuários específicos consegue alcançar objetivos direcionados com eficiência, eficácia e satisfação subjetiva ${ }^{23,24}$. Assim, os aplicativos podem ser de fácil entendimento, eficazes e eficientes para certas pessoas enquanto que, para outras, podem ser inadequados ${ }^{24}$. Em geral, os aplicativos apresentam uma breve descrição com explicações sobre a funcionalidade ${ }^{10}$. Cerca de $80 \%$ dos aplicativos avaliados definem claramente a sua funcionalidade nas informações fornecidas pelos criadores.

\section{Conclusão}

O presente trabalho possibilitou o levantamento e a avaliação de 57 aplicativos sobre ansiedade e depressão em Língua Portuguesa. Os aplicativos foram avaliados com base em critérios definidos para a avaliação de aplicativos de Saúde e saúde mental. Não foram encontrados trabalhos relacionados com o objetivo de avaliar sistematicamente os aplicativos para ansiedade e depressão em Língua Portuguesa. 
Como forma de mitigar possíveis ameaças à validade do estudo, foi adotada uma metodologia para a avaliação sistemática dos aplicativos. Conflitos durante a seleção e avaliação foram discutidos entre os pesquisadores para mitigar o viés pessoal.

Aplicativos com foco na ansiedade e depressão estão se tornando bastante populares. Contudo, os resultados obtidos a partir da avaliação demonstram a necessidade de melhorias em termos de diversos aspectos. A popularização desses aplicativos, bem como a falta de pesquisas relacionadas, demonstra a importância de avaliar os softwares disponíveis.

Constata-se que a maioria dos aplicativos avaliados não possui embasamento científico, carecendo de referências científicas, bem como do uso de técnicas de estudos que comprovem a sua eficácia, pois, quando o aplicativo é baseado em evidências científicas, pode constituir uma estratégia promissora, servindo como fonte de informação e representando uma ferramenta segura para os usuários. Identifica-se, dessa forma, a possibilidade de colaboração entre universidades, centros de pesquisa e projetistas para o desenvolvimento e aprimoramento de aplicativos em Língua Portuguesa com base em evidências científicas.

Observa-se também que a maioria dos aplicativos analisados não é acessível para pessoas com deficiências, apresentando limitações de uso por esse público.

Entre as limitações no desenvolvimento do estudo, destaca-se escassez de aplicativos para iOS em Língua Portuguesa.

Como possibilidade de trabalhos futuros, o presente estudo pode servir como orientação para pesquisadores de diferentes áreas de pesquisa que almejam desenvolver ou aprimorar aplicativos em Língua Portuguesa com foco em ansiedade e depressão, além de trazer uma reflexão sobre a importância desse recurso digital para a população.

Encontra-se em desenvolvimento, um aplicativo denominado QuizAD ${ }^{24}$, para a plataforma Android, que tem como propósito auxiliar no rastreio da ansiedade e depressão, contribuindo para um diagnóstico precoce, com base na Escala Hospitalar de Ansiedade e Depressão (HAD) $)^{25}$.

\section{Referências}

1. Who. Mental Health Atlas 2014. [Internet]. Department of Health: World Health Organization; [Acesso 2019 Set 19]. Disponível em: https://www.who.int/mental_health/evidence/atlas/mental_health_atlas_2014/en/ 
2. Pavan Marina. Design e tecnologia: criação de ferramenta de conscientização sobre depressão [trabalho de conclusão de curso]. Lajeado: Universidade do Vale do Taquari - Univates Centro de Ciências Humanas e Sociais Curso de Design; 2017.

3. Lopes KC da SP, Santos WL dos. Transtorno de ansiedade. Rev Inic Cient Ext [Internet]. $25^{\circ}$ de junho de 2018 [citado $21^{\circ}$ de outubro de 2019];1(1):45-50. Disponível em: https://revistasfacesa.senaaires.com.br/index.php/iniciacaocientifica/article/view/47.

4. Godoy PG, Schutz SM. Plataforma mobile para depressivos: aplicativo Rhope. Cadernos de iniciação científica: $5^{a}$ Jornada de Integração e Iniciação Científica. 2019;3(2).

5. Souza JC de. Depressão, a medicalização, o mercado de antidepressivos e a busca de uma nova ação terapêutica. Trabalho de Conclusão de Curso (Especialização em Gestão da Inovação em Fitomedicamentos) - Instituto de Tecnologia em Fármacos / Farmanguinhos, Fundação Oswaldo Cruz, Rio de Janeiro, 2017. $41 \mathrm{f}$.

6. OMS. Depressão é tema de campanha da OMS para Dia Mundial da Saúde de 2017 [Internet]. Nações Unidas Brasil: Organização Mundial da Saúde (OMS); [atualizado 2016 Dez 19; Acesso 2019 Set 19]. Disponível em:

https://nacoesunidas.org/depressao-e-tema-de-campanha-da-oms-para-diamundial-da-saude-de-2017/.

7. OMS. OMS registra aumento de casos de depressão em todo o mundo; no Brasil são 11,5 milhões de pessoas [Internet]. Nações Unidas Brasil: Organização Mundial da Saúde (OMS); [atualizado 2017 Fev 24; Acesso 2019 Set 19]. Disponível em: https://nacoesunidas.org/oms-registra-aumento-de-casos-de-depressao-emtodo-o-mundo-no-brasil-sao-115-milhoes-de-pessoas/.

8. Gusmão EÉ da S, et al. Esquemas desadaptativos, ansiedade e depressão: proposta de um modelo explicativo. Revista Brasileira de Terapias Cognitivas. 2017;13(1):29-38.

9. Bonafe FSS, Carvalho J de S, Campos JADB. Depressão, ansiedade e estresse e a relação com o consumo de medicamentos. Psicologia, Saúde \& Doenças. 2016;17(2):105-119.

10. Barbosa SS. Funcionalidades para aplicativo de celular à pessoa com comportamento suicida no brasil [trabalho de conclusão de curso]. Florianópolis: Universidade Federal de Santa Catarina Centro de Ciências da Saúde; 2018;109.

11. Henson $P$ et al. Deriving a practical framework for the evaluation of health apps. The Lancet Digital Health. 2019;1(2):52-54.

12. Rocha TAH, et al. "Saúde Móvel: novas perspectivas para a oferta de serviços em saúde." Epidemiologia e Serviços de Saúde 25 (2016): 159-170.

13. Larsen ME; Nicholas J; Christensen H. A Systematic Assessment of Smartphone Tools for Suicide Prevention. Public Library of Science (PLoS) One. 2016; 11(4): 114.

14. Coutinho GL. A Era dos Smartphones: Um estudo exploratório sobre o uso dos Smartphones no Brasil. 2014. 60f., il. Monografia (Bacharelado em comunicação Social). Brasília.

15. Kitchenham B., 2007. Guidelines For Performing Systematic Literature Reviews In Software Engineering. EBSE Technical Report. Keele University \& Department of Computer Science University of Durham.

16. Travassos GH, Santos PSM dos, Mian PG, Neto PGM, Biolchini J. An Environment to Support Large Scale Experimentation in Software Engineering. In: 13th IEEE International Conference on Engineering of Complex Computer Systems (iceccs 
2008) [Internet]. Belfast: IEEE; 2008 [citado 18 de maio de 2020]. p. 193-202. Disponível em: https://ieeexplore.ieee.org/document/4492892/

17. Brereton $P$, Kitchenham BA, Budgen D, Turner M, Khalil M. Lessons from applying the systematic literature review process within the software engineering domain. Journal of Systems and Software. abril de 2007;80(4):571-83.

18. Zaman Sojib Bin et al. Contextos e oportunidades da tecnologia de e-saúde na assistência médica. Revista de Pesquisa e Inovação Médicas. 2017; 1(2): AV1AV4.

19. Mendez CB, Salum NC, Junkes C, Amante IN, Mendez CML. Mobile educational follow-up application for patients with peripheral arterial disease. Rev. Latino-Am. Enfermagem. 2019; 27: e3122. [Acesso 25 out 2019]; Disponível em: http://www.scielo.br/pdf/rlae/v27/pt_0104-1169-rlae-27-e3122.pdf DOI: http://dx.doi.org/10.1590/1518-8345.2693-3122. Out 252019.

20. Formagini TDB, et al. Revisão dos aplicativos de smartphones para cessação do tabagismo disponíveis em língua portuguesa. Cadernos de Saúde Pública 2017; 33 (2): e00178215.

21. Henry S; Lawton. Introduction to Web Accessibility. Disponível em: https://www.w3.org/WAl/fundamentals/accessibility-intro/. Acesso em: 14 Mai. 2020.

22. Barbosa PHM. Análise das Permissões e Violações de Privacidade em Aplicações para Android. (2017). Programa de Graduação em Engenharia da Computação do Centro de Informática da Universidade Federal de Pernambuco.

23. Henry S. Lawton SA-Z; Kevin W. Introduction to Web Accessibility. Disponível em: https://www.w3.org/WAl/fundamentals/accessibility-usability-inclusion/. Acesso em: 14 Mai. 2020.

24. Neto; Machado; Olibario José. Usabilidade da interface de dispositivos móveis: heurísticas e diretrizes para o design. Diss. (2013). Universidade de São Paulo.

25. Santos GB et al. QuizAD: um aplicativo para rastreio inicial de ansiedade e depressão. I Congresso Sergipano Multidisciplinar Abordagens em Saúde, Universidade Federal de Sergipe, 2019.

26. Marcolino JÁM et al. Escala hospitalar de ansiedade e depressão: estudo da validade de critério e da confiabilidade com pacientes no pré-operatório. Rev. Bras Anestesiol, v. 57, n. 1, p. 52-62, 2007. 


\section{Anexos}

Figura 1 - Processo de revisão dos aplicativos.

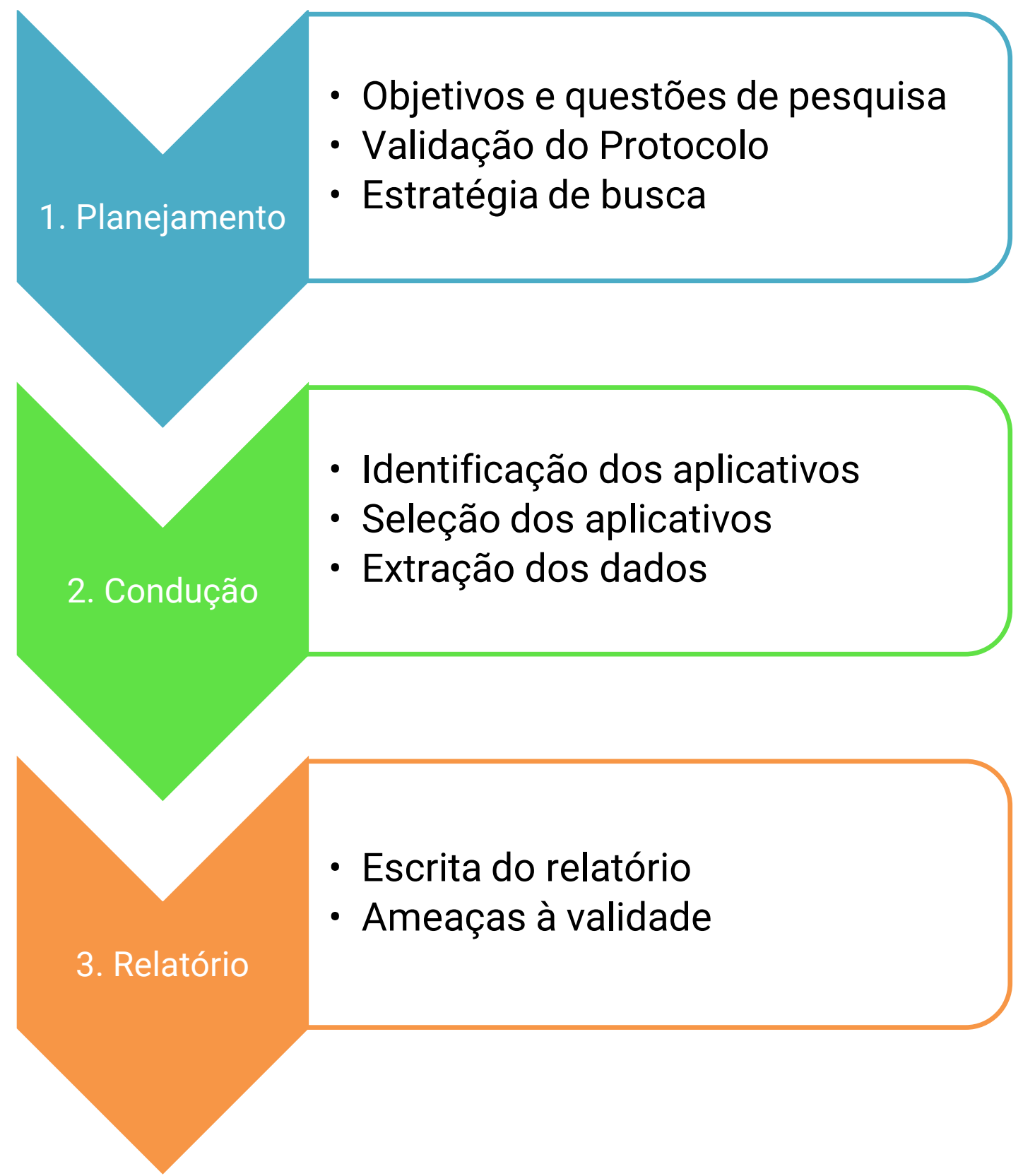

Fonte: Autoria própria. Com base no processo de revisão sistemática ${ }^{17}$. 
Figura 2. Pirâmide de figuras para a estrutura de avaliação de aplicativos.

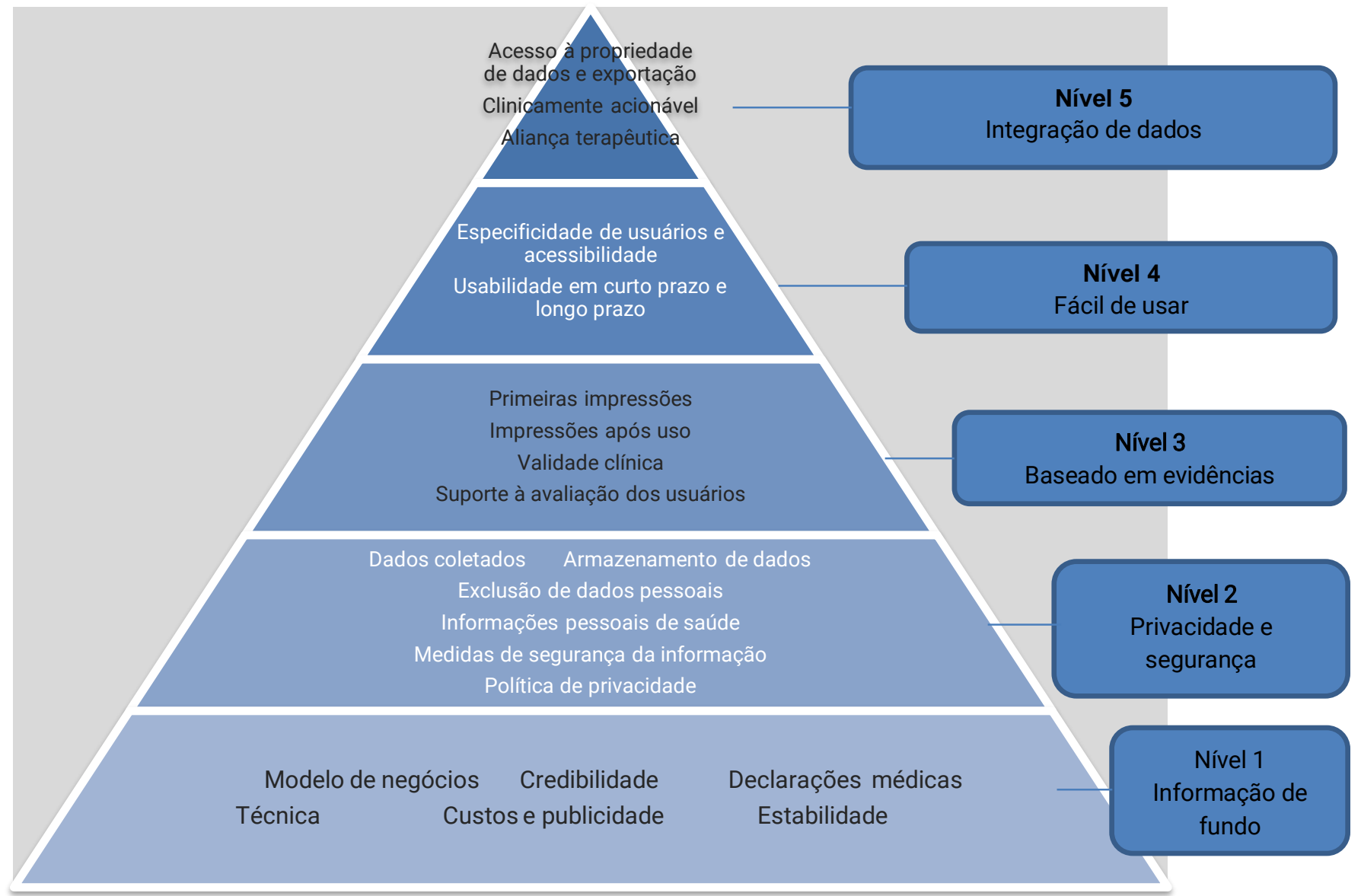

Fonte: Autoria própria. Adaptado de ${ }^{11}$. 
Tabela 1: Aplicativos avaliados.

\begin{tabular}{|c|c|c|c|c|c|c|c|c|c|}
\hline Aplicativo & 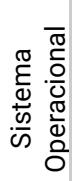 & $\begin{array}{l}\text { Cita fonte de } \\
\text { informações? }\end{array}$ & $\begin{array}{l}\text { Custos } \\
\text { ocultos ou } \\
\text { anúncios? }\end{array}$ & $\begin{array}{l}\text { Precisa de } \\
\text { internet? }\end{array}$ & $\begin{array}{c}\text { Atualizado } \\
\text { nos } \\
\text { últimos } \\
180 \text { dias? }\end{array}$ & $\begin{array}{l}\text { Política de } \\
\text { privacidade? }\end{array}$ & $\begin{array}{l}\text { Pode } \\
\text { excluir } \\
\text { conta? }\end{array}$ & $\begin{array}{l}\text { Bloqueio por } \\
\text { senha ou } \\
\text { criptografia } \\
\text { dos dados? }\end{array}$ & $\begin{array}{c}\text { Funciona } \\
\text { como } \\
\text { prometido? }\end{array}$ \\
\hline $\begin{array}{l}\text { PSY terapia } \\
\text { psicológica } \\
\text { on-line. } \\
\text { Anônimo chat }\end{array}$ & $\begin{array}{l}\frac{0}{0} \\
\frac{0}{\frac{0}{0}} \\
\frac{\mathrm{c}}{<}\end{array}$ & Não & Sim & Sim & Sim & Sim & Sim & Não & Sim \\
\hline $\begin{array}{l}\text { Sons para } \\
\text { dormir- } \\
\text { relaxar e } \\
\text { dormir }\end{array}$ & $\begin{array}{l}\frac{0}{0} \\
\frac{0}{\frac{0}{0}} \\
\frac{\mathrm{c}}{4}\end{array}$ & Não & Sim & Sim & Sim & Não & Sim & Não & Sim \\
\hline $\begin{array}{l}\text { Oração de } \\
\text { cura }\end{array}$ & $\begin{array}{l}\frac{0}{0} \\
\frac{0}{\frac{0}{0}} \\
\frac{2}{4}\end{array}$ & Sim & Sim & Não & Não & Não & Sim & Não & Sim \\
\hline $\begin{array}{l}\text { Mega quiz } \\
\text { discipulado }\end{array}$ & $\begin{array}{l}\frac{0}{0} \\
\frac{0}{\frac{0}{0}} \\
\frac{\mathrm{T}}{4}\end{array}$ & Sim & Sim & Sim & Sim & Sim & Sim & Não & Sim \\
\hline $\begin{array}{l}\text { Frases } \\
\text { depressivas } \\
\text { em português }\end{array}$ & $\begin{array}{l}\frac{0}{0} \\
\frac{0}{0} \\
\frac{2}{4}\end{array}$ & Não & Sim & Não & Não & Sim & Sim & Não & Sim \\
\hline $\begin{array}{l}\text { Alerta para } \\
\text { medicamento } \\
\text { s- lembretes } \\
\text { de remédios }\end{array}$ & $\begin{array}{l}\frac{0}{0} \\
\frac{0}{\frac{0}{0}} \\
\frac{\mathrm{C}}{<}\end{array}$ & Sim & Sim & Não & Sim & Sim & Sim & Não & Sim \\
\hline $\begin{array}{l}\text { Meditação } \\
\text { wemind - } \\
\text { vença a } \\
\text { ansiedade }\end{array}$ & $\begin{array}{l}\frac{0}{0} \\
\frac{0}{0} \\
\frac{1}{4}\end{array}$ & Sim & Sim & Sim & Sim & Sim & Não & Não & Sim \\
\hline $\begin{array}{l}\text { Como } \\
\text { controlar a } \\
\text { ansiedade }\end{array}$ & $\begin{array}{l}\frac{0}{0} \\
\frac{\overline{0}}{0} \\
\frac{\mathrm{c}}{4}\end{array}$ & Não & Sim & Sim & Sim & Não & Sim & Não & Sim \\
\hline Rede do bem & $\begin{array}{l}\frac{7}{0} \\
\frac{0}{0} \\
\frac{0}{4}\end{array}$ & Não & Sim & Não & Não & Sim & Sim & Não & Sim \\
\hline $\begin{array}{l}\text { Psicologia de } \\
\text { bolso }\end{array}$ & $\begin{array}{l}\frac{0}{0} \\
\frac{0}{0} \\
\frac{0}{4}\end{array}$ & Sim & Sim & Não & Sim & Sim & Sim & Não & Sim \\
\hline Psico test & $\begin{array}{l}\frac{70}{0} \\
\frac{0}{0} \\
\frac{0}{4}\end{array}$ & Não & Sim & Sim & Sim & Sim & Sim & Não & Sim \\
\hline
\end{tabular}




\begin{tabular}{|c|c|c|c|c|c|c|c|c|c|}
\hline $\begin{array}{l}\text { Queridos } \\
\text { textos - amor, } \\
\text { poesia e } \\
\text { ansiedade }\end{array}$ & 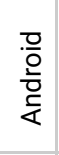 & Não & Sim & Sim & Sim & Não & Não & Não & Sim \\
\hline $\begin{array}{l}\text { Meditopia: } \\
\text { meditação, } \\
\text { ansiedade }\end{array}$ & $\begin{array}{l}\text { 응 } \\
\frac{\overline{0}}{0} \\
\frac{\mathrm{c}}{<}\end{array}$ & Não & Não & Sim & Sim & Sim & Sim & Sim & Não \\
\hline Hepi & & Sim & Não & Não & Sim & Sim & Sim & Não & Sim \\
\hline $\begin{array}{l}\text { Ansiedade e } \\
\text { estresse } \\
\text { diário }\end{array}$ & $\begin{array}{l}: \frac{0}{0} \\
\frac{0}{0} \\
\frac{\mathrm{c}}{<}\end{array}$ & Não & Sim & Sim & Não & Sim & Sim & Não & Sim \\
\hline $\begin{array}{l}\text { Diário: } \\
\text { controle de } \\
\text { humor }\end{array}$ & $\begin{array}{l}: \frac{0}{0} \\
\frac{0}{0} \\
\frac{c}{4}\end{array}$ & Não & Sim & Sim & Sim & Sim & Não & Não & Sim \\
\hline $\begin{array}{l}\text { Teste } \\
\text { ansiedade }\end{array}$ & $\begin{array}{l}\frac{0}{0} \\
\frac{0}{0} \\
\frac{5}{4}\end{array}$ & Sim & Sim & Sim & Não & Sim & Sim & Não & Sim \\
\hline $\begin{array}{l}\text { Ansiedade } \\
\text { (AGALVES) }\end{array}$ & $\begin{array}{l}\frac{0}{0} \\
\frac{0}{0} \\
\frac{0}{4}\end{array}$ & Não & Sim & Sim & Sim & Não & Sim & Não & Sim \\
\hline $\begin{array}{l}\text { Maconha } \\
\text { Brasil }\end{array}$ & $\begin{array}{l}\frac{0}{0} \\
\frac{0}{0} \\
\frac{7}{4}\end{array}$ & Não & Não & Sim & Sim & Sim & Sim & Não & Não \\
\hline $\begin{array}{l}\text { Vivo } \\
\text { meditação - } \\
\text { relaxar e } \\
\text { controlar } \\
\text { ansiedade }\end{array}$ & $\begin{array}{l}\frac{0}{0} \\
\frac{0}{0} \\
\frac{7}{4}\end{array}$ & Não & Não & Sim & Sim & Sim & Sim & Não & Sim \\
\hline $\begin{array}{l}\text { Emoods } \\
\text { diários para } \\
\text { bipolares }\end{array}$ & $\begin{array}{l}\frac{0}{0} \\
\frac{0}{0} \\
\frac{5}{4}\end{array}$ & Não & Não & Sim & Sim & Sim & Não & Não & Não \\
\hline $\begin{array}{l}\text { Como vencer } \\
\text { a depressão }\end{array}$ & $\begin{array}{l}\frac{0}{0} \\
\frac{0}{0} \\
\frac{7}{4}\end{array}$ & Sim & Não & Sim & Sim & Sim & Sim & Não & Sim \\
\hline $\begin{array}{l}\text { Passatempos } \\
\text { inteligentes }\end{array}$ & $\begin{array}{l}\frac{0}{0} \\
\frac{0}{0} \\
\frac{5}{4}\end{array}$ & Sim & Sim & Sim & Sim & Sim & Sim & Não & Sim \\
\hline $\begin{array}{l}\text { Ansiedade e } \\
\text { estresse, } \\
\text { como } \\
\text { combatê-los }\end{array}$ & $\begin{array}{l}\text { 응 } \\
\text { 음 } \\
\text { 둔 }\end{array}$ & Não & Não & Sim & Sim & Sim & Sim & Não & Não \\
\hline
\end{tabular}




\begin{tabular}{|c|c|c|c|c|c|c|c|c|c|}
\hline Pentágono & $\begin{array}{l}\frac{7}{0} \\
\frac{2}{0} \\
\frac{\mathrm{C}}{4}\end{array}$ & Não & Sim & Sim & Sim & Não & Sim & Não & Sim \\
\hline $\begin{array}{l}\text { Root - alívio } \\
\text { do ataque de } \\
\text { pânico e } \\
\text { ansiedade }\end{array}$ & $\begin{array}{l}\frac{0}{0} \\
\frac{1}{0} \\
\frac{7}{4}\end{array}$ & Não & Sim & Sim & Sim & Sim & Sim & Sim & Sim \\
\hline $\begin{array}{l}\text { Monitor de } \\
\text { ansiedade e } \\
\text { humor }\end{array}$ & $\begin{array}{l}\frac{0}{0} \\
\frac{1}{2} \\
\frac{c}{4}\end{array}$ & Não & Sim & Sim & Sim & Sim & Sim & Não & Não \\
\hline $\begin{array}{l}\text { Depressão e } \\
\text { Ansiedade }\end{array}$ & $\begin{array}{l}\text { 응 } \\
\frac{0}{\frac{0}{2}} \\
\frac{\mathrm{c}}{4}\end{array}$ & Não & Sim & Sim & Não & Sim & Sim & Não & Não \\
\hline $\begin{array}{l}\text { Querida } \\
\text { ansiedade }\end{array}$ & $\begin{array}{l}\text { 응 } \\
\frac{\text { 음 }}{\frac{c}{4}}\end{array}$ & Sim & Sim & Sim & Sim & Sim & Sim & Sim & Sim \\
\hline $\begin{array}{l}\text { Cíngulo: } \\
\text { terapia guiada }\end{array}$ & $\begin{array}{l}\text { 음 } \\
\text { 0 } \\
: \frac{0}{0} \\
\text { 음 } \\
\text { 둔 }\end{array}$ & Não & Sim & Sim & Sim & Sim & Sim & Não & Não \\
\hline $\begin{array}{l}\text { Depressão } \\
\text { teste e } \\
\text { exercícios }\end{array}$ & ?. & Não & Sim & Sim & Sim & Sim & Sim & Não & Não \\
\hline $\begin{array}{l}\text { Anxiety e } \\
\text { depression } \\
\text { Mundo } \\
\text { psicólogos }\end{array}$ & 요 & Sim & Sim & Sim & Sim & Sim & Sim & Não & Sim \\
\hline Zung & 으 & Sim & Sim & Não & Não & Não & Não & Não & Sim \\
\hline $\begin{array}{l}\text { Controle e } \\
\text { monitor: } \\
\text { ansiedade, } \\
\text { humor e } \\
\text { autoestima }\end{array}$ & $\begin{array}{l}\frac{0}{0} \\
\frac{1}{0} \\
\frac{c}{4}\end{array}$ & Sim & Sim & Sim & Não & Sim & Sim & Sim & Sim \\
\hline $\begin{array}{l}\text { Avoid - Você } \\
\text { consegue? }\end{array}$ & $\begin{array}{l}\frac{0}{0} \\
\frac{0}{0} \\
\frac{1}{4}\end{array}$ & Não & Sim & Sim & Sim & Sim & Sim & Não & Sim \\
\hline $\begin{array}{l}\text { Lojong: } \\
\text { Meditação e } \\
\text { Mindfulness. } \\
\text { Reduza a } \\
\text { ansiedade }\end{array}$ & $\begin{array}{l}\frac{0}{0} \\
\frac{0}{0} \\
\frac{C}{4}\end{array}$ & Sim & Sim & Sim & Sim & Não & Sim & Sim & Sim \\
\hline $\begin{array}{l}\text { Teste de } \\
\text { depressão (de } \\
\text { Zung) }\end{array}$ & $\begin{array}{l}\frac{0}{0} \\
\frac{1}{0} \\
\frac{c}{4}\end{array}$ & Não & Sim & Sim & Sim & Não & Sim & Não & Não \\
\hline
\end{tabular}




\begin{tabular}{|c|c|c|c|c|c|c|c|c|c|}
\hline $\begin{array}{l}\text { Ombro amigo: } \\
\text { conselhos e } \\
\text { desabafo } \\
\text { anônimo - } \\
\text { Chat }\end{array}$ & $\begin{array}{l}\frac{0}{0} \\
\frac{0}{\frac{0}{0}} \\
\frac{7}{4}\end{array}$ & Não & Sim & Sim & Sim & Sim & Sim & Sim & Sim \\
\hline $\begin{array}{l}\text { Ansiedade e } \\
\text { estresse, } \\
\text { como superá- } \\
\text { los }\end{array}$ & $\begin{array}{l}\frac{0}{0} \\
\frac{0}{0} \\
\frac{0}{4}\end{array}$ & Não & Sim & Sim & Não & Não & Não & Não & Não \\
\hline $\begin{array}{l}\text { Qustodio } \\
\text { controle } \\
\text { parental }\end{array}$ & $\begin{array}{l}\frac{0}{0} \\
\frac{0}{0} \\
\frac{5}{4}\end{array}$ & Não & Sim & Sim & Sim & Sim & Não & Sim & Sim \\
\hline Zen & $\begin{array}{l}\frac{7}{0} \\
\frac{0}{0} \\
\frac{0}{4}\end{array}$ & Não & Sim & Sim & Sim & Sim & Não & Não & Não \\
\hline $\begin{array}{l}\text { Frases de } \\
\text { tristeza }\end{array}$ & $\begin{array}{l}\frac{0}{0} \\
\frac{0}{0} \\
\frac{0}{4}\end{array}$ & Não & Sim & Sim & Não & Sim & Sim & Não & Sim \\
\hline $\begin{array}{l}\text { Documentário } \\
\text { s sobre sauúde } \\
\text { em geral }\end{array}$ & $\frac{\text { 음 }}{\frac{0}{0}}$ & Sim & Sim & Sim & Não & Sim & Sim & Não & Sim \\
\hline $\begin{array}{l}\text { Treinar seu } \\
\text { cérebro- jogos } \\
\text { de raciocínio }\end{array}$ & 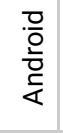 & Sim & Sim & Sim & Sim & Não & Não & Não & Sim \\
\hline $\begin{array}{l}\text { Treinar seu } \\
\text { cérebro - } \\
\text { jogos de } \\
\text { atenção }\end{array}$ & $\begin{array}{l}\frac{0}{0} \\
\frac{0}{0} \\
\frac{2}{4}\end{array}$ & Sim & Sim & Sim & Sim & Não & Não & Não & Sim \\
\hline $\begin{array}{l}\text { Frases de } \\
\text { motivação }\end{array}$ & 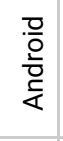 & Não & Sim & Não & Sim & Não & Sim & Não & Sim \\
\hline $\begin{array}{l}\text { Amigo virtual- } \\
\text { novas } \\
\text { amizades e } \\
\text { desabafo } \\
\text { anônimo }\end{array}$ & $\begin{array}{l}\frac{0}{0} \\
\frac{0}{0} \\
\frac{0}{4}\end{array}$ & Não & Sim & Sim & Sim & Não & Sim & Não & Sim \\
\hline $\begin{array}{l}\text { Poder da } \\
\text { mente - } \\
\text { motivação e } \\
\text { lei da atração }\end{array}$ & $\begin{array}{l}\frac{0}{0} \\
\frac{0}{0} \\
\frac{7}{4}\end{array}$ & Não & Sim & Não & Sim & Não & Sim & Não & Sim \\
\hline $\begin{array}{l}\text { Diário: } \\
\text { registro de } \\
\text { humor }\end{array}$ & $\begin{array}{l}\text { 음 } \\
\text { 음 } \\
\text { 둔 }\end{array}$ & Não & Sim & Não & Não & Não & Sim & Não & Sim \\
\hline
\end{tabular}




\begin{tabular}{|c|c|c|c|c|c|c|c|c|c|}
\hline $\begin{array}{l}\text { O que você } \\
\text { prefere? }\end{array}$ & $\begin{array}{l}\frac{0}{0} \\
\frac{0}{0} \\
\frac{9}{4}\end{array}$ & Não & Sim & Não & Sim & Não & Sim & Não & Não \\
\hline Info mental & $\begin{array}{l}\frac{0}{0} \\
\frac{0}{0} \\
\frac{9}{4}\end{array}$ & Sim & Sim & Não & Sim & Não & Sim & Não & Sim \\
\hline $\begin{array}{l}\text { Hipnose } \\
\text { autocura }\end{array}$ & $\begin{array}{l}\frac{0}{0} \\
\frac{0}{0} \\
\frac{0}{4}\end{array}$ & Não & Sim & Sim & Sim & Não & Sim & Não & Sim \\
\hline $\begin{array}{l}\text { Hipnoterapia } \\
\text { hipnotizar } \\
\text { com hipnose }\end{array}$ & $\begin{array}{l}\frac{0}{0} \\
\frac{0}{0} \\
\frac{5}{4}\end{array}$ & Sim & Sim & Sim & Sim & Não & Sim & Não & Não \\
\hline $\begin{array}{l}\text { Mundo } \\
\text { psicólogos - } \\
\text { bem-estar e } \\
\text { psicologia }\end{array}$ & 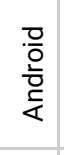 & Sim & Sim & Sim & Sim & Não & Sim & Não & Sim \\
\hline $\begin{array}{l}\text { Castbox: } \\
\text { podcast } \\
\text { gratuito }\end{array}$ & 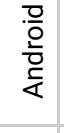 & Sim & Sim & Sim & Sim & Não & Sim & Não & Sim \\
\hline $\begin{array}{l}\text { Mais cor - } \\
\text { cromoterapia }\end{array}$ & 爱 & Sim & Sim & Sim & Sim & Não & Sim & Não & Não \\
\hline $\begin{array}{l}\text { Teste de } \\
\text { personalidade } \\
\text { científica }\end{array}$ & $\begin{array}{l}\frac{0}{0} \\
\frac{0}{0} \\
\frac{0}{2}\end{array}$ & Sim & Sim & Sim & Sim & Não & Sim & Não & Sim \\
\hline
\end{tabular}

Fonte: Autoria própria. 